\title{
Optical Microscopy as a Tool for Studying Sex Differences in Pain and Analgesia
}

\author{
Martin Wessendorf ${ }^{1}$, Nai-Jiang Liu ${ }^{2}$, Emiliya Storman ${ }^{2}$ and Alan Gintzler ${ }^{2}$ \\ ${ }^{1}$ University of Minnesota, Minneapolis, Minnesota, United States, ${ }^{2}$ State University of New York, \\ Downstate, Brooklyn, New York, United States
}

The sensation of pain is one that is experienced by virtually every human being. However, the same stimulus can evoke experiences that vary wildly from individual to individual, and from occasion to occasion. Some of these differences appear to be related to sex. However, the mechanisms that underlie these differences remain unclear. We have examined several different aspects of these differences using a combination of behavioral, biochemical, and light microscopic methods.

Females typically have lower pain thresholds than males. However, the pain thresholds of females progressively increase during pregnancy until plummeting back to their non-pregnant levels immediately after parturition (Cogan and Spinnato, 1986). We studied this phenomenon behaviorally in female rats treated with estrogen and progesterone to simulate the levels experienced in pregnancy. We found that the increase in pain threshold was associated with increased release of the endogenous $\kappa$-opioid agonist dynorphin (DYN), and that the increased pain threshold could be blocked by $\kappa$-opioid receptor antagonists. We hypothesized that increased levels of estrogen were key to this change and examined the lumbosacral spinal cord for neurons expressing DYN and estrogen receptor- $\alpha(\mathrm{ER} \alpha)$. We found that significantly more neurons expressing ER $\alpha$-immunoreactivity (-ir) in the L6 and S1 spinal segments in animals in which pregnancy had been simulated with hormone treatment. In addition, a significantly higher proportion of these neurons also expressed DYN-ir (Figure 1), suggesting that the increase in DYN was induced by the increased expression of estrogen during pregnancy (Gintzler et al. 2008).

Normal cycling of estrogen levels also affects pain thresholds. In female rats, this appears due to an increase in the heterodimerization of $\mu$-opioid receptors and $\kappa$-opioid receptors. We therefore hypothesized that in the spinal cord, estrogen receptors would be coexpressed with both $\mu$-opioid receptors and $\kappa$-opioid receptors, and that $\mu$-opioid receptors and $\kappa$-opioid receptors would also be coexpressed. We found numerous examples of coexpression of ER $\alpha$ with $\mu$-opioid receptors using multicolor fluorescence microscopy (Figure 2). In addition, we were able to follow single cells using serial sectioning and thereby demonstrate single neurons that expressed ER $\alpha, \mu$-opioid receptors, and $\kappa$-opioid receptors. (Liu et al, 2011).

The sex organs appear not to be the only source of circulating estrogen. We and others have observed activity for aromatase (estrogen synthase) in the central nervous system, including in regions of the hypothalamus that project their axons to the pituitary, outside the blood-brain barrier. We hypothesized that hypothalamic neurons expressing aromatase could be retrogradely labeled by tract-tracers injected into the peripheral circulation. When we injected the retrograde tract-tracer Fluoro-Gold (hydroxystilbamidine) into the tail vein, we found many examples of retrogradely labeled neurons that expressed aromatase-ir. This finding suggests that estrogen synthesized in the brain doesn't simply have local effects - the brain may also function as an endocrine organ releasing estrogen systemically (Storman et al, 2018).

We draw several conclusions from these studies. First, the increase in pain threshold observed in pregnancy appears at least in part to be due to DYN being expressed by increased numbers of spinal neurons. Second, m-opioid and $\kappa$-opioid receptors are coexpressed with each other and with ER $\alpha$. Based on our biochemical data, we propose that changing levels of estrogen result in changing degrees of 
heterodimerization of $\mu$ - and k-opioid receptors. Finally, the CNS itself may generate at least some of the estrogen that provokes these effects. These findings provide mechanisms that may underlie, at least in part, sex differences in pain.
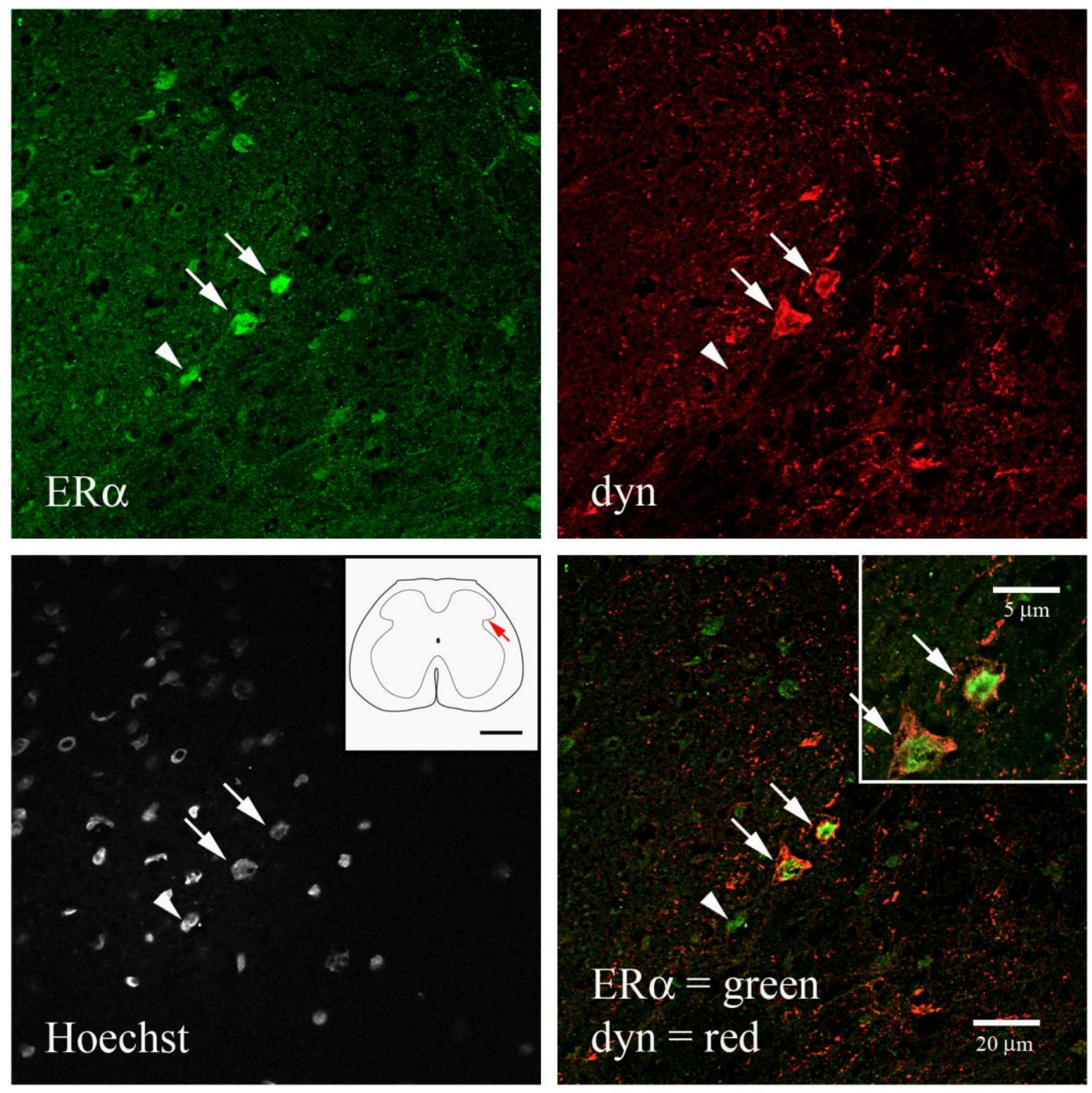

Figure 1. Expression of estrogen receptor $\alpha(E R \alpha)$ by neurons expressing the endogenous opioid dynorphin (DYN) in the superficial dorsal horn of the lower lumbosacral spinal cord. We found that expression of DYN in ER $\alpha$ neurons increased during hormone-simulated pregnancy. Hoechst: Hoechst 33258, a fluorescent stain for cell nuclei. 


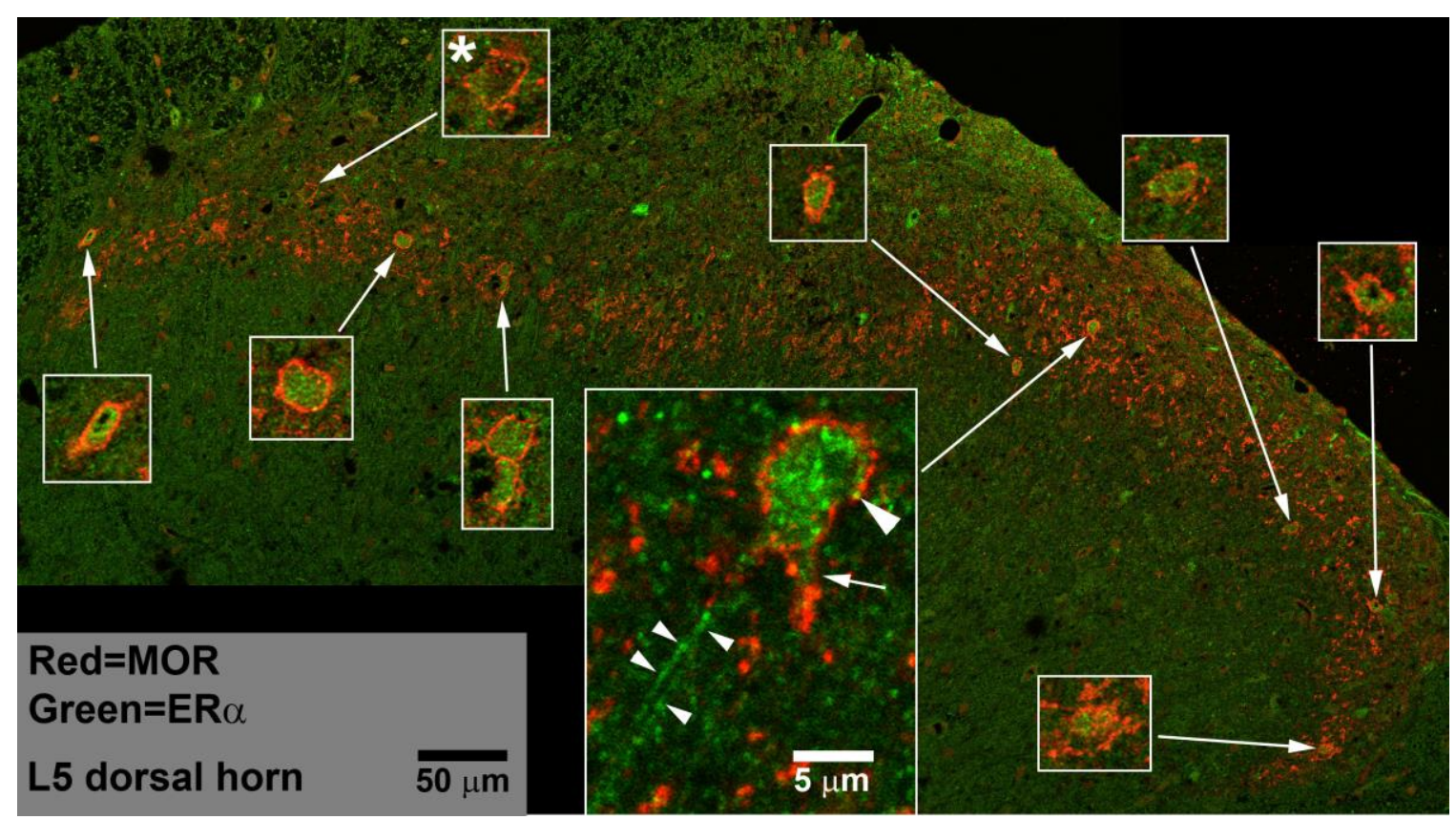

Figure 2. Expression of the $\mu$-opioid receptor by lumbosacral dorsal horn neurons expressing $\mathrm{ER} \alpha$. Expression of ER $\alpha$ by these neurons provides an anatomical substrate for the regulation of opiate analgesia by sex hormones.

\section{References}

Cogan R, Spinnato JA. Pain and discomfort thresholds in late pregnancy. Pain. 1986 Oct;27(1):63-8. Gintzler AR, Schnell SA, Gupta DS, Liu NJ, Wessendorf MW. Relationship of spinal dynorphin neurons to delta-opioid receptors and estrogen receptor alpha: anatomical basis for ovarian sex steroid opioid antinociception. J Pharmacol Exp Ther. 2008 Sep;326(3):725-31

Liu NJ, Chakrabarti S, Schnell S, Wessendorf M, Gintzler AR. Spinal synthesis of estrogen and concomitant signaling by membrane estrogen receptors regulate spinal $\kappa$ - and $\mu$-opioid receptor heterodimerization and female-specific spinal morphine antinociception. J Neurosci. 2011 Aug 17;31(33):11836-45

Storman EM, Liu NJ, Wessendorf MW, Gintzler AR. Physical Linkage of Estrogen Receptor $\alpha$ and Aromatase in Rat: Oligocrine and Endocrine Actions of CNS-Produced Estrogens. Endocrinology. 2018 Jul 1;159(7):2683-2697 